\title{
IoT Based Automatic Control of Sun Tracking Solar Panel for High Power Generation
}

\author{
RuckmaniDivakaran, G. N. Nandini ,N. Pavithra, D. Priya, B. Y. Ramya R. Dharshini \\ Departmentof Electronicsand Communication Engineering \\ Dr.T.Thimmaiah Institute of Technology, Kolar Gold Fields \\ deanedrttit.edu.in
}

\begin{abstract}
The conversion of sun light into electric energy through solar panels is significant compared to other renewable sources. The energy extracted from the solar panel depends on solar light incident on the solar panel, but the constant variation in the sun's position decreases the power generation efficiency. In order to extract maximal energy, the solar panel should face the sunlight at normal angle throughout the day. Solar tracker tilts the panel towards the sun light direction. The automatic sun tracking solar panel will harness a significant amount of energy from available sun light. Single axis type of solar tracker is used which has one degree of freedom of rotation. Closed loop tracking approach is used with LDR's, an ATmega2560 microcontroller and a DC motor forming the principal components of the circuit model. Based on the signals generated from LDR's, microcontroller provides signals to the motor for tilting the solar panel towards the direction of maximum incident sun rays, which will increase the power generation efficiency. The efficiency of the proposed system is $71 \%$. The information regarding status of solar tracking system is shared through IoT.
\end{abstract}

Keywords:Solar tracker, LDR, ATmega2560 Microcontroller, DC motor, IoT.

\section{Introduction}

Conventional power resources like petroleum fuel, lignite and fossil gases are not only getting depleted but also pollute the environment. As energy consumption and demand level continue to rise with rising global population, hence there is a need of filling the gap through renewable energy. The most significant source of renewable energy is solar energy. Photovoltaic panels were introduced to use this solar energy. Solar panel is an array of solar cells arranged in an order it absorbs sun light and converts it into electrical energy. Solar cell is made up of semiconductor substance silicon.The availability of the solar energy is unlimited; harnessing it optimally presents a challenge because of the stationary nature of photovoltaic panels. 


\section{Literature Survey}

This survey explains about the Sun tracking solar panels using various techniques having its own advantages, and disadvantages. It is a review of relevant literature materials in relation to the topic considered. A Kassem and M Hamad [1] proposed "A Microcontroller-based multi-function solar tracking system" a system which aligns the solar panel towards the sun light. The drawback is the proposed system has reasonable efficiency only during summer Sunny day. Khatri V Yas et.al [2] proposed, "Development of Automatic PV Powerpack Servo Based Single Axis Solar Tracking System" a single axis tracker model. The microcontroller code, and servo mechanism is simulated in PROTEOUS7. The system stops tilting during the night. Power generation efficiency is 7.67\%. Protik Kumar Das et.al [3] proposed, "Microcontroller Based Automatic Solar Tracking System with Mirror Booster" a tracking approach where mirror is used as a booster. Stepper motor and photodiode are the main system components. Mirror is used to boost and increase the efficiency.

The system will rotate from north to south and south to north in circular motion. This system is suitable for power generation in large scale. The power generation efficiency is $9 \%$. The drawback is the system is bulky. Aashish et.al [4] proposed, "Sun tracking solar panel with a Maximum PowerPoint tracking" a low cost model. It is a realtime clock model. MPPT is to control the solar panels in a way that allows the solar panels to produce all the capable power. The system uses an electronic hardware circuit and an algorithm [6]. The drawback is that impedence match between solar panel and load circuit is important for increasing the efficiency. Neenu Sharma et.al [5] proposed "An Analysis of Automatic Dual Axis Sun Tracking Solar System" a study of dual axis tracker. Orientation and tilting are the key factors of this paper. The drawback is that mono crystalline PV panel is used in this paper where absorption rate is lesser compared to polycrystalline panels.

\section{System Methodology}

Solar tracking system have three significant components, an input stage with light sensors, a program in embedded software in microcontroller and an output stage with DC motor. Closed loop tracking approach is used with three LDRs, an [7] Arduino with ATmega2560 microcontroller and a DC motor forming the principal components of the circuit model. Single axis type of solar tracking is used. The Fig 1, represent the block diagram of the IoT based automatic sun tracker. Solar tracker orients the payload towards the sunlight direction. Loads are solar panels, lenses reflectors, and optical devices. LDR sensors are used to monitor the sun's position[8],[9]. Output voltage of the LDR's varies in inverse proportion to the intensity of the radiation. These voltages are fed to the ADC. The ADC converts the analog voltages to digital signals and sends the signals to the microcontroller. The microcontroller will perform the necessary arithmetic and logical operations based on the data it received and issues control signals to the DC motor. DC motor will rotate clockwise or anticlockwise based on 
the signals generated from the microcontroller in order to tilt the solar panel towards maximum sun light. Cloud computing is the most important algorithm today.[10][11].

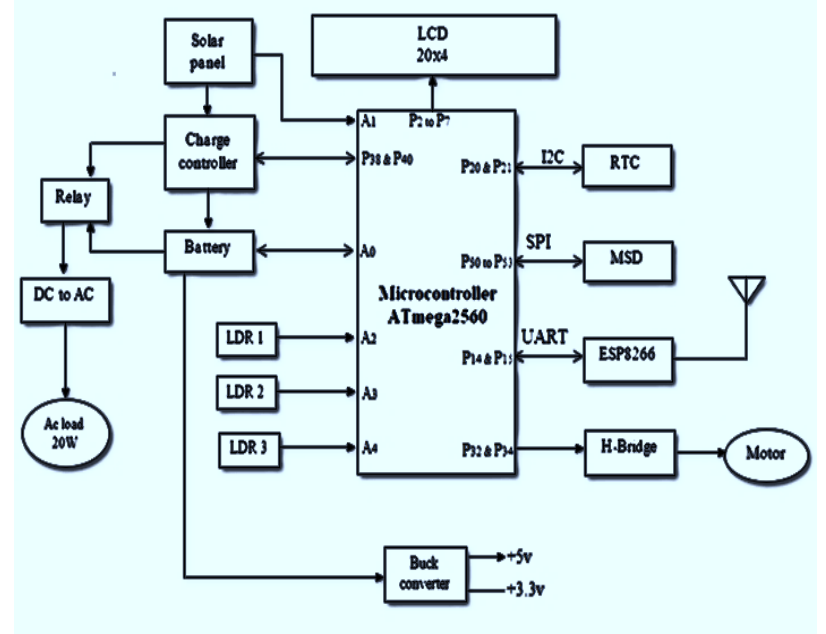

Fig.1: Block Diagram of Sun Tracking Solar Panel

\section{$4 \quad$ Hardware Component}

\subsection{Arduino Mega:}

The Arduino Mega is a development board which is designed for ATmega2560 microcontroller.

\subsection{ATmega2560 Microcontroller}

ATmega2560 is an 8-bit microcontroller. The architecture of this microcontroller is an advanced virtual RISC architecture i.e AVR architecture.ATmega2560 microcontroller is used for controlling the direction of DC gear motor. It has $256 \mathrm{~KB}$ of flash memory for storing code. It is programmed to make decisions based on LDR signals.

\subsection{ESP8266 WIFI Module}

The ESP8266 is a Wi-Fi chip embedded with Transmission control protocol/Internet Protocol (TCP/IP). It is designed for IoT applications. It is compact in design and integrated with Wi-Fi System on Chip. 


\section{Implementation}

Closed loop tracking approach is used with three of LDRs, an Arduino with ATmega2560 microcontroller and a DC motor forming the principal components of the circuit model. Single axis type of solar tracking is used i.e. aligned east-west directions. Two phases of implementation: Hardware implementation and software implementation.

\subsection{Hardware Implementation}

Hardware is the physical aspects of the model. Hardware connections of the electronic circuitry are done to implement the sun tracking system. Mechanical assembly of the solar panel and DC motors are done.

\subsection{Software Implementation}

A set of instructions that enable physical components of the sun tracking system to work in a synchronized way is known as program or code.

Step 1: Code is written and compiled in Arduino IDE with no errors.
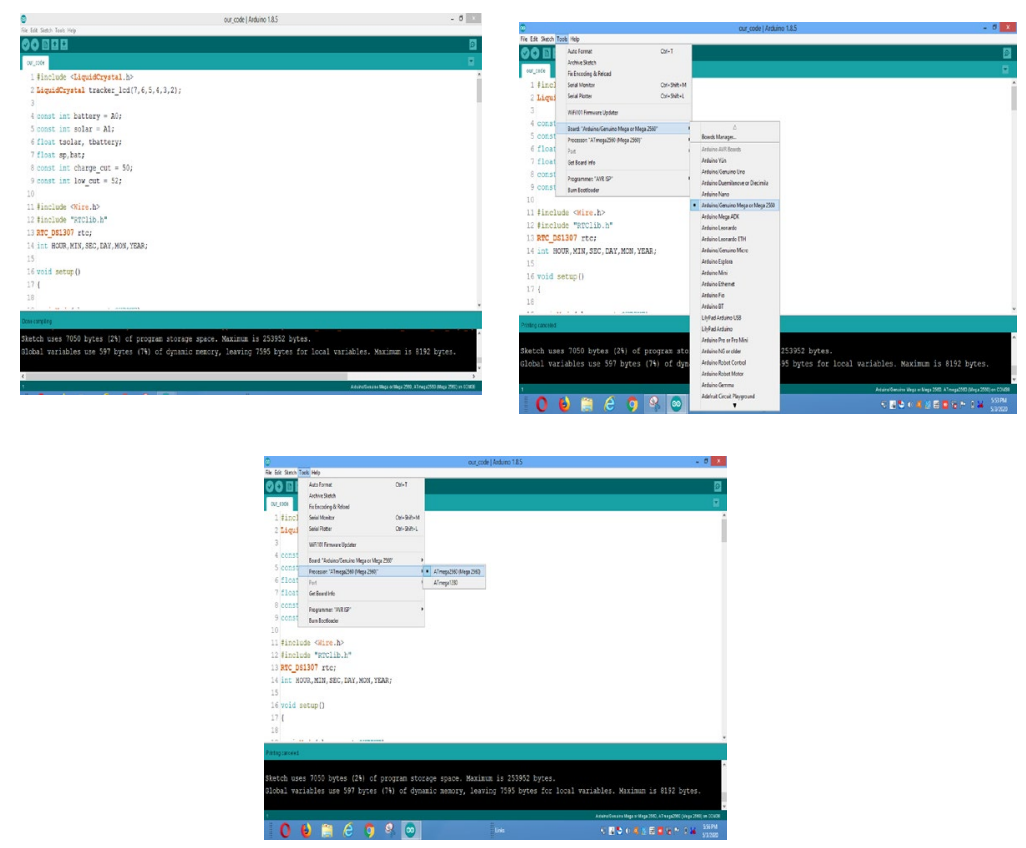


\section{Discussion}

To assess the effectiveness and working of the model, solar panel voltage and battery voltage values are recorded. Table 1 , shows the comparison between solar panel voltage in Volts without tracking and with tracking.

Table 1: Comparison of the solar panel voltage without tracking and with tracking

\begin{tabular}{|c|c|c|c|c|}
\hline $\begin{array}{l}\text { Time } \\
\text { In Hour }\end{array}$ & $\begin{array}{l}\text { Without Track- } \\
\text { ing Solar Panel } \\
\text { Voltage in Volts }\end{array}$ & $\begin{array}{l}\text { Tracking Solar } \\
\text { Panel Voltage in } \\
\text { Volts }\end{array}$ & $\begin{array}{l}\text { Efficiency \% } \\
\text { with tracking }\end{array}$ & $\begin{array}{l}\quad 40 \% \text { Solar panel effi- } \\
\text { ciency }+ \text { efficiency with } \\
\text { tracking }\end{array}$ \\
\hline 8:00 & 12.20 & 15.62 & 28.00 & 68.00 \\
\hline $8: 30$ & 12.40 & 16.12 & 30.00 & 70.00 \\
\hline 9:00 & 12.60 & 16.38 & 30.00 & 70.00 \\
\hline $9: 30$ & 12.70 & 16.51 & 30.00 & 70.00 \\
\hline $10: 00$ & 12.80 & 16.64 & 30.00 & 70.00 \\
\hline $10: 30$ & 12.90 & 16.77 & 30.00 & 70.00 \\
\hline $11: 00$ & 13.00 & 16.90 & 30.00 & 70.00 \\
\hline $11: 30$ & 13.10 & 17.03 & 30.00 & 70.00 \\
\hline $12: 00$ & 13.20 & 17.29 & 31.00 & 71.00 \\
\hline $12: 30$ & 13.30 & 17.42 & 31.00 & 71.00 \\
\hline $1: 00$ & 13.40 & 17.42 & 30.00 & 70.00 \\
\hline $1: 30$ & 13.30 & 17.38 & 30.70 & 70.70 \\
\hline $2: 00$ & 13.30 & 17.38 & 30.70 & 70.70 \\
\hline $2: 30$ & 13.20 & 17.25 & 30.70 & 70.70 \\
\hline $3: 00$ & 13.10 & 17.12 & 30.70 & 70.70 \\
\hline $3: 30$ & 13.10 & 17.12 & 30.70 & 70.70 \\
\hline 4:00 & 13.00 & 16.99 & 30.70 & 70.70 \\
\hline $4: 30$ & 13.00 & 16.99 & 30.70 & 70.70 \\
\hline
\end{tabular}

Example taken for the set of values: 


$$
\begin{gathered}
\text { Efficiency }=\left[\left[\frac{\boldsymbol{V}_{\boldsymbol{W T}}-\boldsymbol{V}_{\boldsymbol{W O T}}}{\boldsymbol{V}_{\boldsymbol{W O T}}}\right] * \mathbf{1 0 0}\right]+\text { Solar panel efficiency } \\
=(17.29-13.20 / 13.20)^{*} 100 \%+40 \%=71 \%,
\end{gathered}
$$

$71 \%$ is peak efficiency Where VWT is solar panel voltage with tracking and VWOT is solar panel voltage without tracking.

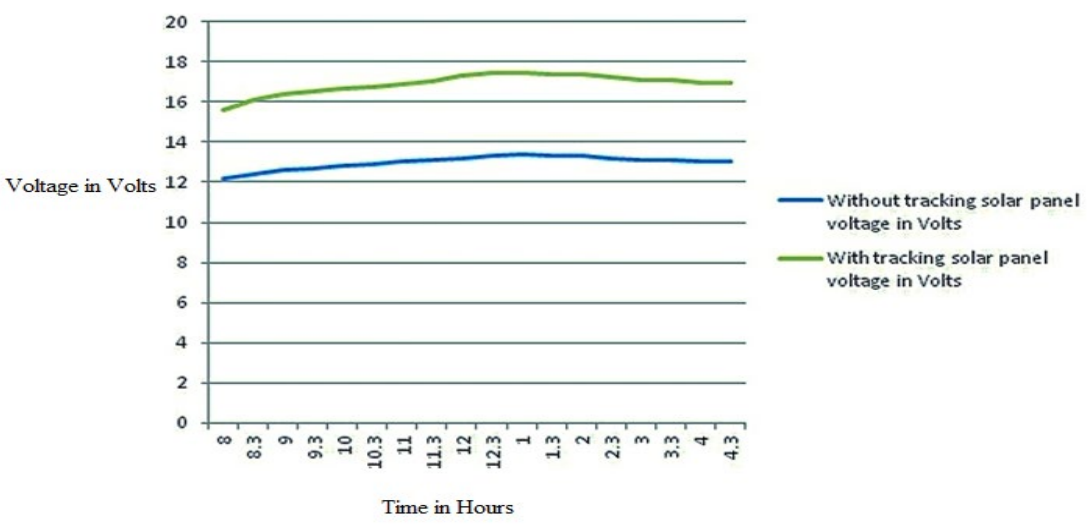

Fig. 2: Comparison of solar panel voltage without tracking and with tracking,

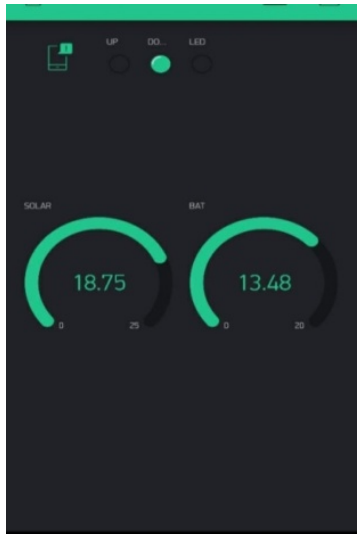

Fig 3: Blynk app output.

Fig 2, shows the graph which compares the solar panel voltage with and without tracking. Blue colored line indicates solar panel voltage value without tracking, and green colored line indicates solar panel voltage value with tracking. The solar panel 
voltage is high with tracking. Fig 3, shows the output of Blynk app. The information regarding solar panel tracking status, solar panel voltage and battery voltage is sent to the authorized person's mobile phone which is installed with Blynk app. This is achieved by using ESP8266 Wi-Fi module which provides internet connectivity to the hardware circuit for monitoring the system from remote place based on IoT.

\section{Conclusion}

The IoT based sun tracking system has been implemented. The system efficiency is $71 \%$. The paper presents a novel controls implementation of the sun tracker and produce high power. Battery's lifetime will increase since charging is halted if the battery is fully charged. ArduinoATmega 2560 microcontroller provides control signals to the DC motor which then rotate to adjust the position of the solar panel. LDR sensors are used which provides more accuracy. The information regarding photovoltaic panel tracking status is sent to the authorized person's mobile phone installed with Blynk app. This makes remotely monitoring of solar plants straight forward and ensures high power generation based on IoT.

\section{REFERENCES}

1. A. Kassem (IEEE Member) and M. Hamad (IEEE Member), "A Microcontroller-Based Multi-Function Solar Tracking System", Conference Paper · May 2011

2. KhyatiVyas, Dr. Sudhir Jain, "Development of Automatic PV Power Pack Servo Based Single Axis Solar Tracking System", IOSR Journal of Electrical and Electronics Engineering (IOSR-JEEE) e-ISSN: 2278-1676, p-ISSN: 2320-3331, Volume 10, Issue 1 Ver. I (Jan - Feb. 2015), PP 07-10 www.iosrjournals.org

3. Protik Kumar Das1, Mir Ahasan Habib1, Mohammed Mynuddin, "Microcontroller Based Automatic Solar Tracking System with Mirror Booster", DOI 10.11648/j.ijrse.20150404.11

4. AashishTiwari, Mayuri, PrajktaShewate, Waghmare Professor, "SunTracking Solar Panel with Maximum Power Point Tracking”, DOI 10.4010/2016.581 ISSN 23213361 (c) 2016 IJESC, Research Article Volume 6 Issue No.3

5. Neenu Sharma, Brijbhushan Sharma, "An Analysis of Automatic Dual Axis Sun Tracking Solar System", International Journal of Innovative Research in Electrical, Electronics, Instrumentation and Control Engineering ISO 3297:2007 Certified Vol. 4, Issue 12, December 2016

6. Kumar, S.S., Ahmed, S.T., Vigneshwaran, P. et al. "Two phase cluster validation approach towards measuring cluster quality in unstructured and structured numerical datasets". J Ambient Intell Human Comput (2020). https://doi.org/10.1007/s12652-020-02487-w

7. Ahmed, S.T., Sankar, S. \& Sandhya, "M. Multi-objective optimal medical data informatics standardization and processing technique for telemedicine via machine learning approach". J Ambient Intell Human Comput (2020). https://doi.org/10.1007/s12652-02002016-9

8. S. S. Thouheed Ahmed, K. Thanuja, N. S. Guptha and S. Narasimha, "Telemedicine approach for remote patient monitoring system using smart phones with an economical hardware kit," 2016 International Conference on Computing Technologies and Intelligent 
Data Engineering (ICCTIDE'16), Kovilpatti, 2016, pp. 1-4, doi: 10.1109/ICCTIDE.2016.7725324

9. K. D. Singh and S. T. Ahmed, "Systematic Linear Word String Recognition and Evaluation Technique," 2020 International Conference on Communication and Signal Processing (ICCSP), Chennai, India, 2020, pp. 0545-0548, doi: 10.1109/ICCSP48568.2020.9182044

10. K. Vijayakumar, S. Suchitra and P. Swathi Shri, "A secured cloud storage auditing with empirical outsourcing of key updates", Int. J. Reasoning-based Intelligent Systems, Vol. 11, No. 2, 2019.

11. K. Vijayakumar, Chokkalingam Arun, "Integrated cloud-based risk assessment model for continuous integration", Int. J. Reasoning-based Intelligent Systems", Vol. 10, Nos. 3/4, 2018. 